\title{
The Impact of the Tracer Size and the Temporal Gap between Images in the Extraction of Atmospheric Motion Vectors
}

\author{
JAVIER GARCÍA-PEREDA \\ AEMET, Madrid, Spain \\ RÉGIS BORDE \\ EUMETSAT, Darmstadt, Germany
}

(Manuscript received 6 November 2013, in final form 22 May 2014)

\begin{abstract}
The goal of this paper is to show the impact of the tracer size and the temporal gap between images in atmospheric motion vector (AMV) extraction schemes. A test has been performed using NWC SAF/High Resolutions Winds AMV software for different configurations with a tracer size varying between $8 \times 8$ and $40 \times 40$ pixels and a temporal gap between images varying between 5 and $90 \mathrm{~min}$. AMVs have been extracted for four different MSG/SEVIRI channels (HRVIS, VIS0.8, WV6.2, and IR10.8) over the European and Mediterranean area for a 6-month period (January-June 2010). The AMV performances have been tested against radiosonde winds and ECMWF model analysis winds.

The results show a small impact of the tracer size on the number of valid AMVs, which is, however, more significant for clear air AMVs, and a significant impact of the temporal gap between images. The largest number of valid AMVs has been found in general for a temporal gap of $5 \mathrm{~min}$ for the 1-km pixel scale and for a temporal gap of $10 \mathrm{~min}$ for the 3-km pixel scale. Results also show a decrease of the mean AMV speed and the normalized BIAS (NBIAS) with larger tracer sizes, and a relatively small impact of the temporal gap on these parameters. Finally, the results show minimum values of the normalized root-mean-square vector difference (NRMSVD) for intermediate temporal gaps between 15 and $30 \mathrm{~min}$ with a relatively small impact of the tracer size on this parameter.
\end{abstract}

\section{Introduction}

Atmospheric motion vectors (AMVs) are derived from tracking clouds or water vapor features in consecutive satellite images. They are the only upper wind observations with good global coverage for the tropics and midlatitudes, especially over the large ocean areas, and they constitute a significant part of the observation data assimilated in numerical weather prediction (NWP) models.

The first step of the AMV extraction scheme is to identify a feature in an initial image that can be tracked in consecutive satellite images. A small target box containing enough contrast with a specified tracer size in pixels is used to characterize such features. To derive the motion, the next step is to locate the position that best

Corresponding author address: Javier García-Pereda, AEMET, Leonardo Prieto Castro 8, E-28040 Madrid, Spain. E-mail: jgarciap@aemet.es; regis.borde@eumetsat.int corresponds to this target box in a later image. A crosscorrelation method can be used to do the matching, as defined by Schmetz et al. 1993. Wind guess information coming from an NWP model can be used to define the location of the search area in the later image before the matching. Using the wind guess permits reduction in the size of the search area and the computing time necessary to derive the AMVs, but this process has not been used in the framework of this study because it can have an impact on the extracted AMVs (Borde and García-Pereda, 2014).

The next step in the process is to assign a pressure value to the derived AMVs. This step is recognized to be the most significant source of error in the AMV extraction process. Several height assignment schemes are used to account for opaque clouds, semitransparent clouds, broken clouds, multilayered clouds, and low level clouds. Borde and Dubuisson (2010) showed that several sources of errors are introduced in the height assignment including the sensitivity of the methods 


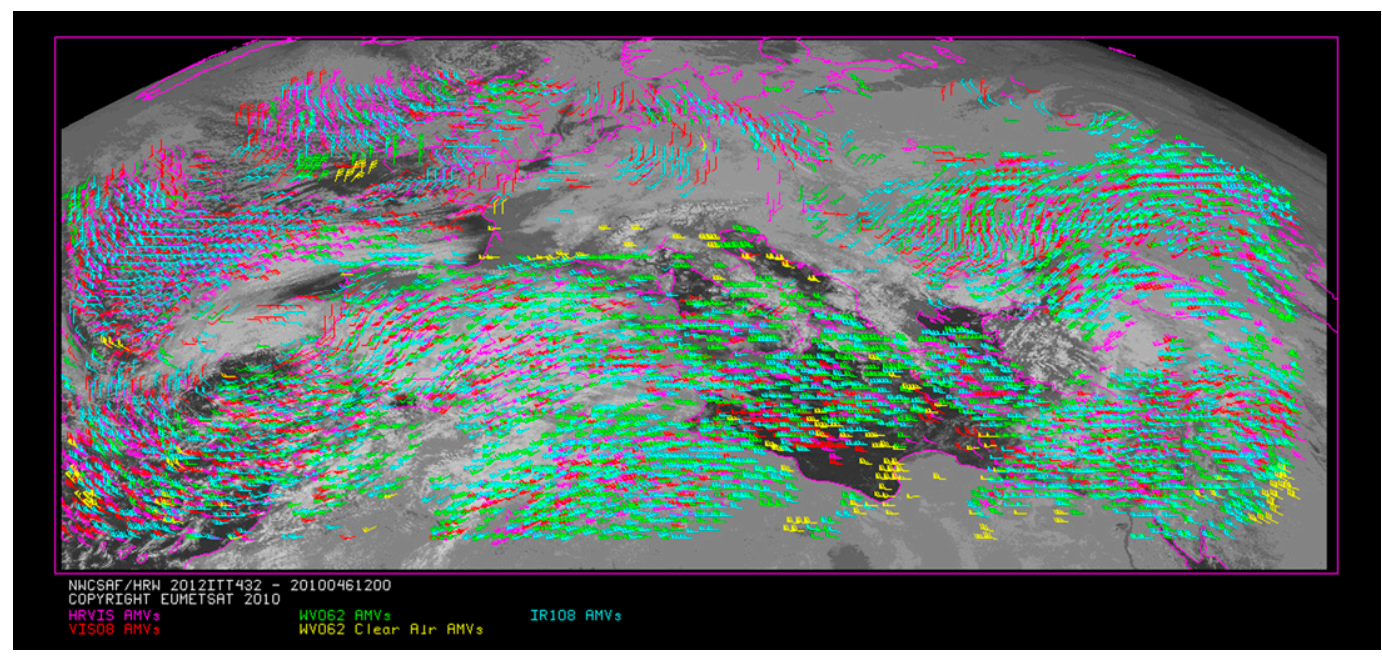

FIG. 1. Display of AMVs over the study region for 1200 UTC 15 Feb 2010 with a $24 \times 24$ pixel tracer size and a 15-min temporal gap between images.

used to local atmospheric parameters, like the cloud depth or the cloud microphysics. Height assignment methods include the water vapor intercept method (Schmetz et al. 1993) and the $\mathrm{CO}_{2}$ slicing method (Menzel et al. 1983).

AMVs have been assimilated in NWP global model for a long time. The recent evolution toward regional models needs assimilation of smaller-scale observations to improve the forecast. Several ways have been investigated to try to extract smaller-scale wind information by using rapid scan imagery or smaller tracer sizes.

There is an obvious link between the spatial scale of the tracked feature and its lifetime. This link is studied in this paper, considering different tracer sizes and different temporal gaps between images in the AMV extraction. The first part of this paper describes the AMV extraction software used and the conditions applied during this study. The later parts show the impact of both parameters on the AMV software performance and discuss the results.

\section{General description of the data}

The High Resolution Winds AMV extraction software (SAFNWC/MSG HRW) used in this study has been developed by AEMET (the Spanish National Weather Service) in the framework of the NWC SAF (the Satellite Application Facility on support to NoWCasting and very short range forecasting). It provides high density sets of AMVs from MSG/SEVIRI images. HRW v3.2, released to users in February 2012, allows the extraction of AMVs using seven different MSG/SEVIRI channels (HRVIS, VIS0.6, VIS0.8, IR10.8, IR12.0, WV6.2, and WV7.3). It includes the cross-correlation contribution (CCC) height assignment method (Borde and Oyama, 2008), implemented operationally at EUMETSAT in September 2012 (Borde et al. 2014), to select the pixels used to calculate the AMV altitude. The EUMETSAT Quality Indicator method (Holmlund, 1998 ) is used to define the quality of the AMV data. For more information, a complete "Algorithm theoretical basis document" (García-Pereda, 2013a) and "Validation report" (García-Pereda, 2013b) for SAFNWC/ MSG HRW software are available at NWC SAF website at www.nwcsaf.org.

AMVs have been extracted from Meteosat-8 MSG/ SEVIRI rapid scan high resolution visible (HRVIS), visible $0.8 \mu \mathrm{m}$ (VIS0.8), infrared $10.8 \mu \mathrm{m}$ (IR10.8), and water vapor $6.2 \mu \mathrm{m}$ (WV6.2) channels, over the European and Mediterranean region $(772 \times 1856 \mathrm{MSG} / \mathrm{SEVIRI}$ low resolution pixels centered in $40.5^{\circ} \mathrm{N}, 11.1^{\circ} \mathrm{E}$, as shown in Fig. 1), for 132 days between January and June 2010, considering three consecutive images finishing at 1200 UTC each day. For this study, 50 parallel runs of NWC SAF/HRW software have been performed, considering five different tracer sizes $(8 \times 8,16 \times 16,24 \times 24$, $32 \times 32$ and $40 \times 40$ pixels) and ten different temporal gaps between images $(5,10,15,20,25,30,45,60,75$ and $90 \mathrm{~min}$ ). The same gridded location of tracers, with a line and element separation of 40 pixels, has been considered in all experiments.

AMVs are extracted using two pairs of images. The second pair provides the final AMV while the first pair is used for temporal quality check. Different search area sizes are used for each configuration to ensure that tracer displacements (i.e., speeds) up to $300 \mathrm{~km} \mathrm{~h}^{-1}$ $\left(83 \mathrm{~m} \mathrm{~s}^{-1}\right)$ per component can be detected. These search area sizes take into account the nominal resolution of 
the images $(1$ or $3 \mathrm{~km})$, the value of the tracer size and the temporal gap between images. For example, for a 5-min temporal gap and a tracer size of $8 \times 8$ pixels, a search area size of $26 \times 26$ pixels is used for the VIS0.8, IR10.8, and WV6.2 low resolution cases, and a search area size of $58 \times 58$ pixels is used for the HRVIS high resolution case. For a 90-min temporal gap and a tracer size of $40 \times 40$ pixels, a search area size of $340 \times 340$ pixels is used for the low resolution cases, and a search area size of $940 \times 940$ pixels is used for the high resolution case. With this configuration, the computational cost of all experiments has been around 18 days of continuous running on an Intel Linux Virtual server, inside a BladeFrame BF400 S2, Xeon IA-32E 4way $2400 \mathrm{MHz}$, with 8-GB RAM and Linux RHEL Server 5.3 Operating System.

The AMV pressure level defined using the CCC height assignment method keeps a direct link between the tracking step and the calculation of the AMV altitude, using the individual contribution to the cross correlation of each pixel inside the target box (Büche et al. 2006). For this process and cloudy AMVs, the CCC method uses the cloud top pressure calculated for each cloudy pixel by the SAFNWC/MSG cloud top temperature and height product. Cloudy pixels are defined by the SAFNWC/MSG cloud type product. The AMV altitude is then the average pressure of the pixels selected by the CCC method, weighted by their individual contribution to the correlation. The pressure level of clear air AMVs has been set using the CCC method together with the pixel brightness temperatures in the water vapor channel to define an AMV brightness temperature; the AMV brightness temperature values are then converted to pressure values through interpolation to the NWP model forecast temperature levels.

The SAFNWC/MSG cloud top pressure retrieval for opaque clouds is based on the window 10.8- and 12.0- $\mu \mathrm{m}$ brightness temperatures and RTTOV radiance simulations. For semitransparent clouds it is based on the radiance ratioing version of the $\mathrm{CO}_{2}$ slicing technique (Menzel et al. 1983) and the water vapor intercept method (Schmetz et al. 1993), as defined in the "Algorithm theoretical basis document for Cloud products (CMa, CT, CTTH)" (Derrien and Le Gléau, 2013). This document is also available at www.nwcsaf.org.

A quality index (QI) with forecast contribution is assigned to each AMV through a quality control process that considers spatial consistency with neighboring winds in the same image, temporal consistency with winds in the previous image, and consistency with the NWP wind component fields (Holmlund, 1998). Only AMVs that have a QI larger than $85 \%$ and a correlation coefficient larger than $80 \%$ are considered in the framework on this study. A QI without forecast contribution has not been considered because it was not calculated in HRW v3.2 software.

NWP profiles needed for the SAFNWC/MSG cloud and AMV product extraction were obtained from the 0000 UTC ECMWF NWP model forecast, having a grid resolution of $0.125^{\circ}$. The data contain 12 vertical levels $(1000,925,850,700,500,400,300,250,200,150,100$, and $70 \mathrm{hPa}$ ) of geopotential, wind components, and air and surface temperature.

The AMVs have been compared and validated against the 1200 UTC ECMWF NWP model wind analysis (MA) and collocated radiosonde (RS) wind observations, following the CGMS criteria defined at the Third International Winds Workshop (Menzel 1996). These criteria are commonly used by all AMV producers to monitor the quality of AMV algorithms: a horizontal separation AMV/RS less than $150 \mathrm{~km}$ and a vertical pressure difference AMV/RS less than $25 \mathrm{hPa}$.

Radiosonde observations are sparse and only a small proportion of the total number of AMVs, especially in some configurations with very long temporal gaps and very large tracer sizes, can be compared to radiosonde winds. To avoid a possible lack of representativeness, these ones have only been compared to NWP model analysis winds.

\section{The effect of the temporal gap between images and the tracer size in the calculation of AMVs}

The effect of the temporal gap between the initial tracer image and the later tracking image is studied considering 5-, 10-, 15-, 20-, 25-, 30-, 45-, 60-, 75-, and 90-min values. A very long gap of $90 \mathrm{~min}$ has been considered in this study as it compares to the extraction of AMVs from low orbit satellites over polar areas where the gap between the consecutive images is close to $100 \mathrm{~min}$. Therefore, specific problems linked to such a very long gap have to be considered. The effect of the tracer size is studied considering $8 \times 8,16 \times 16,24 \times 24$, $32 \times 32$, and $40 \times 40$ pixel values, respectively.

The variation of the number of AMVs produced as a function of the tracer size is presented in Fig. 2 for just the 5-, 15-, 30-, and 60-min temporal gaps in order to maintain clarity in the figure. Therefore, some comments below may refer to results obtained for configurations that have not been plotted in Fig. 2.

The maximum number of AMVs is obtained for a 5-min temporal gap using the high resolution HRVIS images and for a 10-min temporal gap using the low resolution VIS0.8, IR10.8, and WV6.2 images when small tracer sizes are used. Longer temporal gaps of 15 to $20 \mathrm{~min}$ are needed for IR10.8 and WV6.2 images 


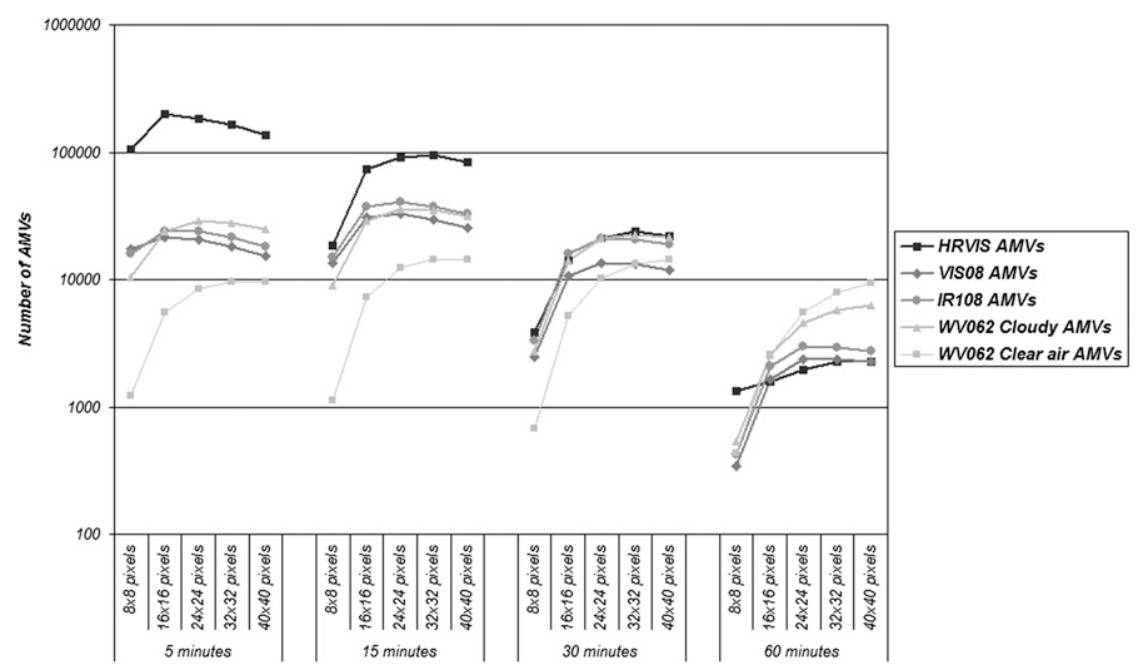

FIG. 2. Variation of the number of AMVs with the tracer size for the cases using a temporal gap between images of $5,15,30$, and $60 \mathrm{~min}$.

when large tracer sizes are used. For longer temporal gaps, significant reductions in the number of cloudy AMVs occur. Compared with the maximum number of AMVs retrieved, $4 \%-16 \%$ of HRVIS AMVs and $12 \%-68 \%$ of the rest of cloudy AMVs remain for a temporal gap of $30 \mathrm{~min}$; only around $2 \%$ of HRVIS AMVs and $2 \%-20 \%$ of the rest of cloudy AMVs are present for a 60-min temporal gap.

Results in Fig. 2 also show that the maximum number of AMVs is related to progressively larger tracer sizes when longer temporal gaps are used. For low resolution visible and infrared cases, the maximum number of AMVs is generally produced for tracer sizes between $16 \times 16$ and $24 \times 24$ pixels. For water vapor cases, the maximum number of AMVs occurs for larger tracer sizes between $24 \times 24$ and $40 \times 40$ pixels; $40 \times 40$ pixels specially when considering clear air AMVs. With the high resolution HRVIS channel, the maximum is in most cases found for $32 \times 32$ pixels.

The number of AMVs extracted by the algorithm is related to the lifetime of the features tracked. If the shape of a feature changes significantly between two images, it cannot be tracked in the later image and so the corresponding AMV cannot be extracted. Not surprisingly, the results for all cloudy AMVs show that the lifetime of the features is longer when they are larger. Mostly the large features can be tracked using long temporal gaps, but the small features have disappeared or have changed their shape too much. This circumstance occurs especially when the smallest tracer size of $8 \times 8$ pixels is considered.

This illustrates that the low contrast and entropy present in a very small target box often prevents the algorithm identifying a good tracer and following it accurately in a series of images. Such reduction of the number of good AMVs when using smaller tracer sizes and longer temporal gaps has been shown by several studies, such as Sohn and Borde (2008) using MTSAT1R/IR10.8 images with several configurations between $8 \times 8$ and $48 \times 48$ pixel tracer sizes and 15-30-min temporal gaps, or more recently Shimoji (2012) using MTSAT-1R/IR10.8 images with several configurations between $5 \times 5$ and $33 \times 33$ pixel tracer sizes and 5-30-min gaps.

This effect has also been studied by Borde and GarcíaPereda (2014) considering the impact of the wind guess in the tracking step of AMV extraction. Their results show that an important number of the good quality AMVs cannot be extracted without the help of the wind guess when using small tracer sizes, especially for long temporal gaps. This result means that although more AMVs can be extracted using the wind guess for small tracer sizes, many of these AMVs contain significant model information.

Considering the different MSG/SEVIRI channels, the lifetime of the features used for the AMV calculation is progressively longer for HRVIS, VIS0.8, IR10.8, and WV6.2 channels, especially when larger tracer sizes are considered. The much shorter lifetime of the cloudiness features in the HRVIS AMVs is related to their much smaller size in kilometers. The longer lifetime of the cloudiness features in the IR10.8 and WV6.2 AMVs is related to the longer persistence in time of the cloudiness brightness temperature features compared to cloudiness reflectance features. The WV6.2 clear air AMVs are based on the tracking of large humidity features that have a longer lifetime. Comparing with the maximum number of AMVs extracted, more than $52 \%$ of clear air AMVs are retained for a $30-\mathrm{min}$ gap, and more than $34 \%$ of 


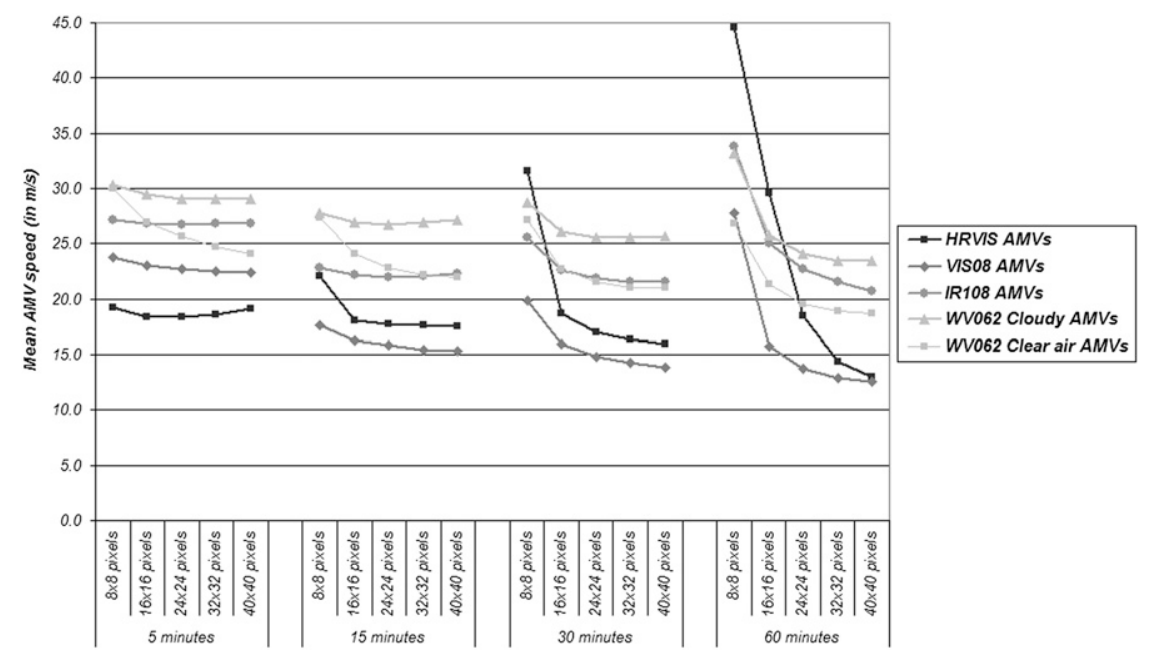

FIG. 3. Variation of the mean AMV speed $\left(\mathrm{m} \mathrm{s}^{-1}\right)$ with the tracer size for the cases using a temporal gap between images of 5, 15, 30 and $60 \mathrm{~min}$.

AMVs for a $60-m i n$ gap. Considering for example all AMVs together for the different channels with a tracer size of 24 pixels, the number of WV6.2 clear air AMVs is only about $3 \%$ of the total for a 5-min gap, but around $49 \%$ of the total for a 90 -min gap. Here it is also necessary to take into account that the smoother and less defined shapes shown by WV6.2 clear air AMVs need generally larger tracer sizes to better define the features and optimize the AMV calculation.

In this study, the mean tracking correlation of the AMVs in each experiment has also been found, for all channels, to be smaller for longer temporal gaps. Anyhow the features defined in the tracers can change their shape significantly even considering short temporal gaps, making their tracking difficult. However, if the features are stable for at least $15-20 \mathrm{~min}$, their tracking in later images is easier because their shape does not change very much thereafter.

Considering 5-10-min gaps, the mean correlation is smaller for small tracer sizes than for large tracer sizes, showing that large tracer sizes are easier to track. Larger mean correlation values are also needed to get good AMV tracking when small tracer sizes and long temporal gaps are considered. This also illustrates increased difficulty tracking small features when the time difference is great. The correlation has to be greater to ensure that the tracking process works correctly.

Figure 3 shows the mean AMV speed in meters per second corresponding to the experiments plotted in Fig. 2. A minimum mean AMV speed is found for progressively longer temporal gaps when large tracer sizes are used. An exception occurs with the clear air AMVs, for which this parameter has always a minimum value for the longest gap of $90 \mathrm{~min}$. The increase in the mean AMV speed is largest for long temporal gaps if small tracer sizes and cloudy AMVs are considered. The differences in the mean AMV speed with the tracer size for the different MSG/SEVIRI channels are smaller when large tracer sizes are used. Very large mean AMV speeds are shown for very long temporal gaps between 60 and $90 \mathrm{~min}$ when small tracer sizes are used.

A general reduction of the mean AMV speed with larger tracer sizes is also seen in all cases in Fig. 3. This variation is very significant for the longest temporal gaps. The explanation is related to a better persistence of the smaller tracers when related to stronger winds, and a better persistence of the larger tracers when related to weaker winds. Sohn and Borde (2008) also observed this behavior.

Note in Fig. 3 that the behavior of the curves is very different for long and short temporal gaps, and especially for HRVIS and VIS0.8 channels. In these cases the mean AMV speeds become extreme when small temporal gaps are used. This result must be considered together with the corresponding results of Fig. 2, which show that the number of AMVs is really reduced for such cases. This means that very specific AMV types are extracted using small tracer sizes and long temporal gaps. They are faster because they are on average located at higher levels in the atmosphere.

The analysis of the validation errors [bias (BIAS) and root-mean-square vector difference (RMSVD)] is based on normalized values (NBIAS and NRSMVD), to eliminate their dependence on the mean AMV speed for each experiment.

Tables 1 and 2 show the NBIAS against radiosonde winds and NWP model analysis winds, respectively, for the different configurations. The behavior is very similar 
TABLE 1. Variation of the normalized bias (NBIAS) against radiosonde winds for all configurations and MSG/SEVIRI channels, with different tracer sizes between $8 \times 8$ pixels and $40 \times 40$ pixels and temporal gaps between 5 and $90 \mathrm{~min}$.

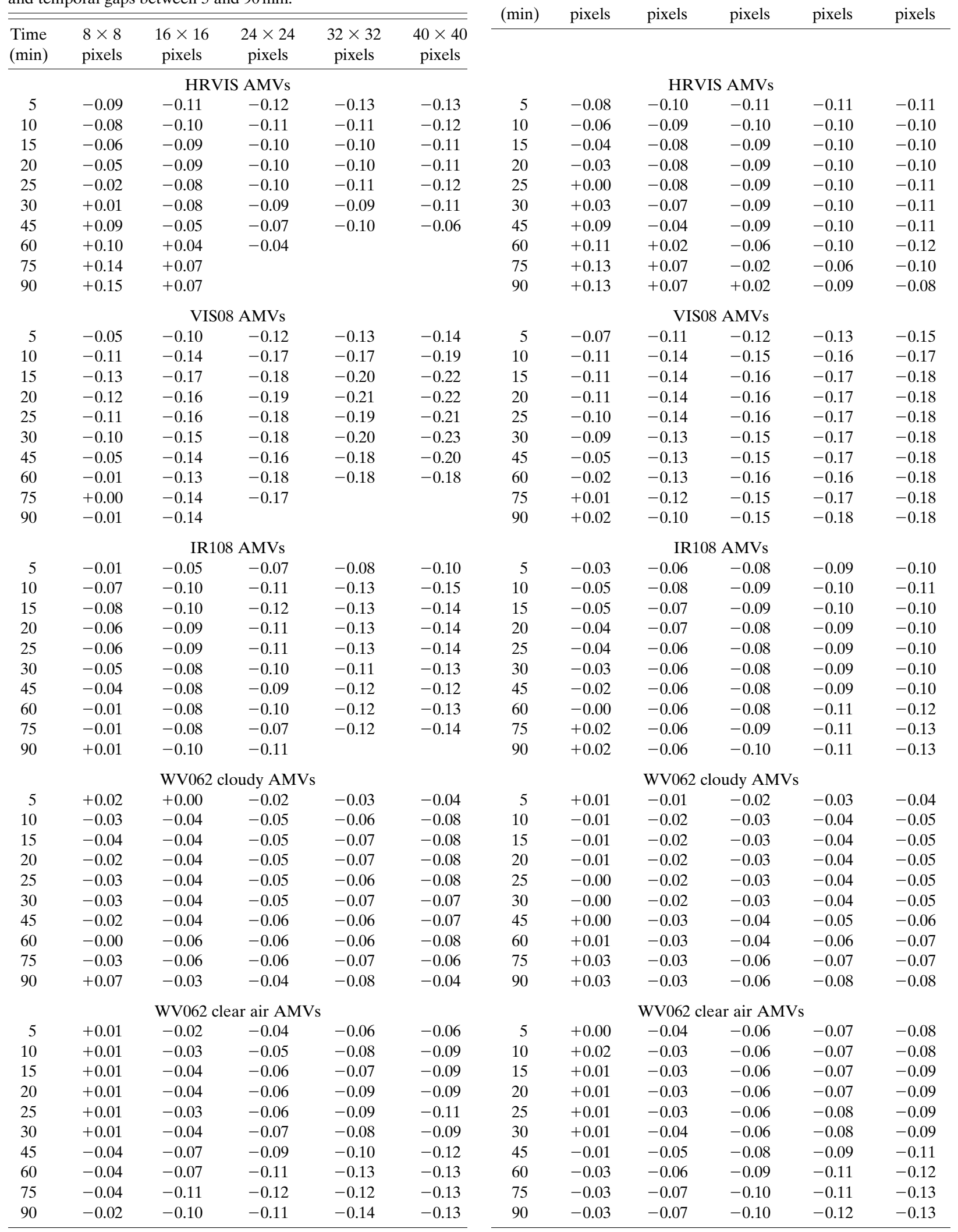

TABLE 2. As in Table 1, but for the normalized bias (NBIAS) against ECMWF NWP model analysis winds.

\begin{tabular}{cccccc}
\hline \hline Time & $8 \times 8$ & $16 \times 16$ & $24 \times 24$ & $32 \times 32$ & $40 \times 40$ \\
$(\min )$ & pixels & pixels & pixels & pixels & pixels \\
\hline
\end{tabular}

VIS08 AMVs

IR108 AMVs

$\begin{array}{llll}-0.07 & -0.09 & -0.10 & -0.10\end{array}$

$\begin{array}{llll}-0.06 & -0.08 & -0.09 & -0.10\end{array}$

$\begin{array}{llll}-0.06 & -0.08 & -0.09 & -0.10\end{array}$

$-0.06-0.08-0.11-0.12$

WV062 cloudy AMVs

WV062 clear air AMVs 
TABLE 3. As in Table 1, but for the normalized root-mean-square vector difference (NRMSVD) against radiosonde winds.

\begin{tabular}{|c|c|c|c|c|c|c|c|c|c|c|c|}
\hline $\begin{array}{l}\text { Time } \\
(\mathrm{min})\end{array}$ & $\begin{array}{l}8 \times 8 \\
\text { pixels }\end{array}$ & $\begin{array}{c}16 \times 16 \\
\text { pixels }\end{array}$ & $\begin{array}{c}24 \times 24 \\
\text { pixels }\end{array}$ & $\begin{array}{c}32 \times 32 \\
\text { pixels }\end{array}$ & $\begin{array}{c}40 \times 40 \\
\text { pixels }\end{array}$ & $\begin{array}{l}\text { Time } \\
(\mathrm{min})\end{array}$ & $\begin{array}{l}8 \times 8 \\
\text { pixels }\end{array}$ & $\begin{array}{c}16 \times 16 \\
\text { pixels }\end{array}$ & $\begin{array}{c}24 \times 24 \\
\text { pixels }\end{array}$ & $\begin{array}{c}32 \times 32 \\
\text { pixels }\end{array}$ & $\begin{array}{c}40 \times 40 \\
\text { pixels }\end{array}$ \\
\hline \multicolumn{6}{|c|}{ HRVIS AMVs } & \multicolumn{6}{|c|}{ HRVIS AMVs } \\
\hline 5 & 0.44 & 0.42 & 0.41 & 0.41 & 0.39 & 5 & 0.31 & 0.30 & 0.29 & 0.29 & 0.28 \\
\hline 10 & 0.41 & 0.39 & 0.39 & 0.38 & 0.37 & 10 & 0.30 & 0.27 & 0.26 & 0.26 & 0.25 \\
\hline 15 & 0.42 & 0.39 & 0.37 & 0.37 & 0.36 & 15 & 0.33 & 0.26 & 0.25 & 0.25 & 0.25 \\
\hline 20 & 0.45 & 0.38 & 0.37 & 0.36 & 0.37 & 20 & 0.38 & 0.27 & 0.25 & 0.25 & 0.25 \\
\hline 25 & 0.47 & 0.39 & 0.37 & 0.37 & 0.37 & 25 & 0.42 & 0.27 & 0.25 & 0.26 & 0.26 \\
\hline 30 & 0.50 & 0.39 & 0.37 & 0.36 & 0.37 & 30 & 0.44 & 0.29 & 0.26 & 0.26 & 0.26 \\
\hline 45 & 0.54 & 0.42 & 0.44 & 0.40 & 0.44 & 45 & 0.47 & 0.38 & 0.32 & 0.30 & 0.29 \\
\hline 60 & 0.53 & 0.50 & 0.43 & & & 60 & 0.49 & 0.43 & 0.39 & 0.36 & 0.37 \\
\hline 75 & 0.54 & 0.53 & & & & 75 & 0.50 & 0.45 & 0.44 & 0.46 & 0.44 \\
\hline 90 & 0.58 & 0.52 & & & & 90 & 0.51 & 0.46 & 0.46 & 0.46 & 0.40 \\
\hline \multicolumn{6}{|c|}{ VIS08 AMVs } & \multicolumn{6}{|c|}{ VIS08 AMVs } \\
\hline 5 & 0.45 & 0.44 & 0.44 & 0.44 & 0.44 & 5 & 0.36 & 0.35 & 0.35 & 0.35 & 0.34 \\
\hline 10 & 0.46 & 0.45 & 0.46 & 0.45 & 0.46 & 10 & 0.35 & 0.35 & 0.35 & 0.35 & 0.35 \\
\hline 15 & 0.46 & 0.46 & 0.47 & 0.47 & 0.48 & 15 & 0.33 & 0.34 & 0.34 & 0.35 & 0.35 \\
\hline 20 & 0.45 & 0.45 & 0.47 & 0.47 & 0.49 & 20 & 0.32 & 0.33 & 0.34 & 0.35 & 0.35 \\
\hline 25 & 0.45 & 0.46 & 0.46 & 0.47 & 0.48 & 25 & 0.33 & 0.32 & 0.34 & 0.34 & 0.34 \\
\hline 30 & 0.46 & 0.45 & 0.47 & 0.48 & 0.51 & 30 & 0.35 & 0.33 & 0.34 & 0.35 & 0.35 \\
\hline 45 & 0.50 & 0.48 & 0.48 & 0.51 & 0.50 & 45 & 0.43 & 0.37 & 0.36 & 0.36 & 0.37 \\
\hline 60 & 0.51 & 0.51 & 0.54 & 0.53 & 0.54 & 60 & 0.47 & 0.44 & 0.40 & 0.38 & 0.40 \\
\hline 75 & 0.54 & 0.57 & 0.57 & & & 75 & 0.49 & 0.48 & 0.45 & 0.44 & 0.42 \\
\hline 90 & 0.50 & 0.57 & & & & 90 & 0.49 & 0.49 & 0.50 & 0.48 & 0.45 \\
\hline \multicolumn{6}{|c|}{ IR108 AMVs } & \multicolumn{6}{|c|}{ IR108 AMVs } \\
\hline 5 & 0.43 & 0.41 & 0.41 & 0.40 & 0.41 & 5 & 0.32 & 0.31 & 0.30 & 0.29 & 0.29 \\
\hline 10 & 0.42 & 0.42 & 0.40 & 0.40 & 0.40 & 10 & 0.27 & 0.26 & 0.26 & 0.26 & 0.26 \\
\hline 15 & 0.40 & 0.40 & 0.39 & 0.39 & 0.39 & 15 & 0.25 & 0.24 & 0.24 & 0.24 & 0.24 \\
\hline 20 & 0.39 & 0.39 & 0.38 & 0.38 & 0.39 & 20 & 0.24 & 0.23 & 0.23 & 0.24 & 0.23 \\
\hline 25 & 0.38 & 0.38 & 0.38 & 0.38 & 0.39 & 25 & 0.25 & 0.23 & 0.23 & 0.23 & 0.24 \\
\hline 30 & 0.38 & 0.38 & 0.38 & 0.38 & 0.38 & 30 & 0.27 & 0.23 & 0.23 & 0.23 & 0.23 \\
\hline 45 & 0.41 & 0.39 & 0.39 & 0.38 & 0.38 & 45 & 0.34 & 0.26 & 0.25 & 0.25 & 0.26 \\
\hline 60 & 0.46 & 0.41 & 0.40 & 0.41 & 0.37 & 60 & 0.39 & 0.32 & 0.29 & 0.29 & 0.29 \\
\hline 75 & 0.49 & 0.46 & 0.43 & 0.42 & 0.45 & 75 & 0.44 & 0.38 & 0.35 & 0.34 & 0.34 \\
\hline 90 & 0.46 & 0.46 & 0.44 & & & 90 & 0.44 & 0.41 & 0.37 & 0.37 & 0.38 \\
\hline \multicolumn{6}{|c|}{ WV062 cloudy AMVs } & \multicolumn{6}{|c|}{ WV062 cloudy AMVs } \\
\hline 5 & 0.43 & 0.40 & 0.39 & 0.38 & 0.38 & 5 & 0.29 & 0.28 & 0.27 & 0.26 & 0.26 \\
\hline 10 & 0.40 & 0.40 & 0.38 & 0.36 & 0.36 & 10 & 0.23 & 0.22 & 0.22 & 0.22 & 0.21 \\
\hline 15 & 0.39 & 0.37 & 0.39 & 0.38 & 0.35 & 15 & 0.21 & 0.21 & 0.21 & 0.20 & 0.20 \\
\hline 20 & 0.37 & 0.38 & 0.37 & 0.35 & 0.35 & 20 & 0.22 & 0.20 & 0.20 & 0.20 & 0.20 \\
\hline 25 & 0.36 & 0.36 & 0.35 & 0.35 & 0.36 & 25 & 0.23 & 0.20 & 0.20 & 0.20 & 0.20 \\
\hline 30 & 0.37 & 0.36 & 0.35 & 0.35 & 0.35 & 30 & 0.24 & 0.21 & 0.20 & 0.21 & 0.21 \\
\hline 45 & 0.41 & 0.37 & 0.37 & 0.37 & 0.37 & 45 & 0.31 & 0.24 & 0.23 & 0.23 & 0.23 \\
\hline 60 & 0.46 & 0.40 & 0.38 & 0.39 & 0.38 & 60 & 0.36 & 0.28 & 0.26 & 0.26 & 0.27 \\
\hline 75 & 0.47 & 0.43 & 0.40 & 0.40 & 0.42 & 75 & 0.42 & 0.33 & 0.32 & 0.32 & 0.32 \\
\hline 90 & 0.51 & 0.45 & 0.42 & 0.47 & 0.42 & 90 & 0.44 & 0.38 & 0.36 & 0.36 & 0.35 \\
\hline \multicolumn{6}{|c|}{ WV062 clear air AMVs } & \multicolumn{6}{|c|}{ WV062 clear air AMVs } \\
\hline 5 & 0.44 & 0.43 & 0.42 & 0.40 & 0.39 & 5 & 0.29 & 0.30 & 0.30 & 0.30 & 0.30 \\
\hline 10 & 0.44 & 0.43 & 0.43 & 0.41 & 0.40 & 10 & 0.26 & 0.27 & 0.28 & 0.28 & 0.28 \\
\hline 15 & 0.45 & 0.42 & 0.42 & 0.41 & 0.40 & 15 & 0.26 & 0.26 & 0.27 & 0.27 & 0.27 \\
\hline 20 & 0.44 & 0.42 & 0.41 & 0.40 & 0.39 & 20 & 0.27 & 0.26 & 0.27 & 0.27 & 0.27 \\
\hline 25 & 0.45 & 0.43 & 0.42 & 0.39 & 0.39 & 25 & 0.28 & 0.26 & 0.27 & 0.27 & 0.27 \\
\hline 30 & 0.47 & 0.41 & 0.40 & 0.38 & 0.38 & 30 & 0.30 & 0.27 & 0.27 & 0.27 & 0.27 \\
\hline 45 & 0.46 & 0.44 & 0.41 & 0.42 & 0.40 & 45 & 0.33 & 0.29 & 0.29 & 0.29 & 0.28 \\
\hline 60 & 0.44 & 0.46 & 0.41 & 0.42 & 0.40 & 60 & 0.35 & 0.32 & 0.30 & 0.30 & 0.30 \\
\hline 75 & 0.48 & 0.43 & 0.43 & 0.43 & 0.41 & 75 & 0.37 & 0.34 & 0.32 & 0.32 & 0.31 \\
\hline 90 & 0.51 & 0.45 & 0.43 & 0.44 & 0.43 & 90 & 0.38 & 0.35 & 0.33 & 0.33 & 0.33 \\
\hline
\end{tabular}

TABLE 4. As in Table 1, but for the normalized root-mean-square vector difference (NRMSVD) against NWP model analysis winds.

WV062 cloudy AMVs 
for both types of reference winds, although the NBIAS is nearer to zero against NWP winds. The NBIAS is negative with generally slight increases with longer temporal gaps when large tracer sizes are used. For small tracer sizes the variations in NBIAS are largest, becoming close to zero and even positive for cloudy AMVs derived from long temporal gap imagery, and for clear air AMVs derived from short temporal gap imagery.

Tables 1 and 2 also show that a more negative NBIAS is seen using larger tracer sizes for all satellite channels. This behavior shows that the mean displacement of the smaller features is in general in better agreement with the mean atmospheric wind. In several cases this trend turns the NBIAS from positive to negative values when the tracer size is larger, especially considering the cases with the longest temporal gaps. The behavior is very similar for both types of reference winds.

Tables 3 and 4 show the NRMSVD against radiosonde winds and NWP model analysis winds for the different experiments. The behavior is again very similar against both types of reference winds, although the parameter is smaller against NWP winds. The minimum NRMSVD value occurs for gaps between 15 and $20 \mathrm{~min}$ for the high resolution channel AMVs, and between 25 and $30 \mathrm{~min}$ for the low resolution channel AMVs. The minimum NRSMVD value against NWP winds is between 0.20 for the WV6.2 Cloudy AMVs and 0.34 for the VIS0.8 AMVs. It increases progressively for longer temporal gaps up to 0.33 for the WV6.2 clear air AMVs and 0.51 for the HRVIS AMVs against NWP winds. The increment is more important for the cloudy AMVs than for the clear air AMVs, which in the comparison get the best NRMSVD for the longest temporal gap. This is caused by the generally longer temporal stability of the humidity features in the clear air AMVs respect to that of the cloudiness features in the cloudy AMVs.

A general reduction of the NRMSVD with larger tracer sizes is also seen in Tables 3 and 4, except in a few cases using short temporal gaps with VIS0.8 or WV6.2 clear air channels for which the impact of the tracer size in the NRMSVD is not significant. The impact on the NRMSVD is largest when long temporal gaps (over $30 \mathrm{~min}$ ) and small tracer sizes (up to $16 \times 16$ pixels) are used. The behavior is again very similar for both types of reference winds.

These results are in good agreement with Shimoji (2012), who showed that the AMV tracking accuracy is degraded decreasing the tracer size or increasing the temporal gap between images. They are also in agreement with Bresky et al. (2012), who obtained a less negative BIAS with smaller tracer sizes, using SEVIRI/IR10.8 images with several configurations between $5 \times 5$ and
$21 \times 21$ pixel tracer sizes and 5-30-min temporal gaps. Their conclusions are similar to results shown in Tables 1 and 2, except for the specific case using a 5-min temporal gap.

Bresky et al. (2012) and Sohn and Borde (2008) also showed a larger RMSVD with smaller tracer sizes, which is in good agreement to results presented in Tables 3 and 4. However, Cho and $\mathrm{Ou}$ (2010) found the opposite result, defining the optimum tracer sizes for AMV calculation considering MTSAT-1R/IR10.8 and MTSAT-1R/ VIS images.

A separate study has been done for this paper, considering the same number of AMVs at the exactly same latitude/longitude localizations for the different configurations. Similar results (not shown) have been obtained: slower mean AMV speeds for larger tracer sizes, and for longer temporal gaps when large tracer sizes are considered; more negative NBIAS values for both larger tracer sizes and longer temporal gaps; minimum NRMSVD values for intermediate temporal gaps, and smaller NRMSVD values for larger tracer sizes. Therefore, the results presented in this paper are inherent to the AMV characteristics in each configuration and not dependent on the specific AMV data used.

In summary the results show that short gaps between 5 and 15 min provide a larger number of AMVs, while intermediate gaps between 15 and $30 \mathrm{~min}$ provide the best validation statistics. Selecting the gap between images for an optimum calculation of AMVs requires a balance to be struck between these two considerations.

Additionally, a larger number of AMVs is calculated with a smaller tracer size. And the mean AMV speed shows a better agreement with the mean radiosonde or NWP winds when the tracer size is small. Nevertheless, it looks like there are limits in the use of the tracer size, and that the use of very small $8 \times 8$ tracer sizes deteriorates the AMV production, decreasing the quality of the extracted AMVs because the tracking becomes very noisy. The results also show that the AMV calculation has smaller errors when larger tracer sizes are used, because they better define the feature to be tracked and thus avoid relating two different features in the initial and later images. A balance is then to be found when selecting the tracer size for an optimum calculation of AMVs.

\section{Discussion}

This paper illustrates the impact of the tracer size and temporal gap between images used for AMV extraction, using the NWC SAF/High Resolution Winds AMV software. Results show an impact of both tracer size and temporal gap on the number on AMVs extracted, 
on the average speed of the AMVs and on their performances against NWP model analysis wind fields and radiosonde wind observations. Results are in general in good agreement with tests performed in the past, but the study is extended to larger sets of tracer sizes and temporal gaps.

The large target boxes contain generally good contrast and entropy to select a good tracer in the first image and to follow it in the later images. Therefore, the larger the tracer size, the easier the matching. But the tracer size is also linked to the size and lifetime of the selected feature. Larger tracer sizes reveal the motion of larger scale features that have a longer lifetime and that can be tracked over a longer period. There are then subtle relationships between the tracer size, the temporal gap between consecutive images, the size and lifetime of the feature tracked, and the quality of the tracking. The impact of the tracer size/temporal gap configuration may be sometimes positive for some of the studied parameters like the number of good AMVs, or the accuracy of the tracking or the performance against other reference winds, and sometimes negative. As explained above, the results show that none of the studied configurations optimize all these criteria at the same time. For example, better NRMSVD values are obtained using very large tracer sizes, but a smaller number of AMVs is then extracted. Therefore, the search of an optimized configuration to be used operationally is not easy, and it implies to find a balance between all these different impacts.

Results of this study show that the best option for the AMV calculation is to reduce the temporal gap between images to $10 \mathrm{~min}$ when possible, or to the nearest longer value when this one is not available. This implies the use of the nominal temporal gap for most currently operating or near future meteorological geostationary satellites: a temporal gap between 10 and $30 \mathrm{~min}$.

Indeed, the number of cloudy AMVs extracted using a 15-min gap is at least $50 \%$ larger than when using gaps of 30 min or longer, while the differences in the average NBIAS and NRMSVD are smaller than $10 \%$. The use of a 10-min gap increases additionally at least $20 \%$ the number of AMVs for the different tracer sizes compared to the use of a 15-min gap, while the change in the average NBIAS and NRSMVD is very small, less than $4 \%$ in both cases.

Considering longer gaps like $1 \mathrm{~h}$, used operationally for example with COMS and MTSAT satellites in the full disk, the results of this study show a significantly lower quality, with $65 \%$ reductions in the number of AMVs and up to 50\% higher NRMSVD values.

Results obtained using a gap smaller than 10 min, like the one nominally defined in rapid scan configuration with current MSG and future MTG satellites, in the CONUS region with future GOES-R satellites, or in the small rapid scan regions with future Himawari 8/9 and GEO-KOMPSAT-2 satellites, show a more noticeable $15 \%$ increase in the NRMSVD, compared to the results obtained using a 10-min temporal gap. Therefore, a separation of at least 10 min between the initial image and the later image to be tracked seems to be optimum.

The use of $16 \times 16$ to $24 \times 24$ pixel tracer sizes appears to be the best options that allow extracting a larger number of cloudy AMVs with good statistics. The $16 \times 16$ pixel configuration has slightly better statistics but extracts less AMVs than the $24 \times 24$ pixel configuration. If a separate processing of water vapor clear air AMVs is an option, a larger tracer size of $40 \times 40$ pixels is recommended to better track their smoother and larger humidity features.

A tracer size of $32 \times 32$ pixels is recommended to optimize the results when the pixel resolution is smaller, around $1 \mathrm{~km}$, certainly in relationship to the shorter lifetime of the corresponding smaller tracers that cannot be tracked so easily with a 15-min temporal gap. This resolution is for example used in the current MSG, GOES-N, MTSAT, COMS, and $F Y$-2 high resolution visible bands, in the future MTG and GOES-R visible and near infrared bands, and in the future Himawari 8/9, GEOKOMPSAT-2 and $F Y-4$ visible bands.

Acknowledgments. Javier García-Pereda and AEMET acknowledge EUMETSAT for the financial funding it has provided for their contribution to this study. The authors also acknowledge Philip Watts for his review of the text of the paper.

\section{REFERENCES}

Borde, R., and R. Oyama, 2008: A direct link between feature tracking and height assignment of operational atmospheric motion vectors. Proc. Ninth Int. Winds Workshop, Annapolis, MD, EUMETSAT, Publ. 51. [Available online at http:// www.eumetsat.int/website/wcm/idc/idcplg?IdcService $=$ GET $_{-}$ FILE\&dDocName $=$ PDF_CONF_P51_S3_13_BORDE_V\& RevisionSelectionMethod $=$ LatestReleased $\&$ Rendition $=$ Web.] , and P. Dubuisson, 2010: Sensitivity of atmospheric motion vectors height assignment methods to semitransparent cloud properties using simulated Meteosat- 8 radiances. J. Appl. Meteor. Climatol., 49, 1205-1218, doi:10.1175/2010JAMC2352.1.

— tracking of atmospheric motion vectors. J. Atmos. Oceanic Technol., 31, 458-467, doi:10.1175/JTECH-D-13-00105.1.

- M. Doutriaux-Boucher, G. Dew, and M. Carranza, 2014: A direct link between feature tracking and height assignment of operational EUMETSAT atmospheric motion vectors. J. Atmos. Oceanic Technol., 31, 33-46, doi:10.1175/ JTECH-D-13-00126.1. 
Bresky, W., J. Daniels, A. Bailey, and S. Wanzong, 2012: New methods toward minimizing the slow speed bias associated with atmospheric motion vectors. J. Appl. Meteor. Climatol., 51, 2137-2151, doi:10.1175/JAMC-D-11-0234.1.

Büche, G., H. Karbstein, A. Kummer, and H. Fischer, 2006: Water vapor structure displacements from cloud-free Meteosat scenes and their interpretation for the wind field. J. Appl. Meteor. Climatol., 45, 556-575, doi:10.1175/JAM2343.1.

Cho, H.-J., and M.-L. Ou, 2010: Determining optimal conditions for mesoscale AMV. Proc. 10th Int. Winds Workshop, Tokyo, Japan, EUMETSAT, Publ. 56. [Available online at http:// www.eumetsat.int/website/wcm/idc/idcplg? IdcService $=$ GET_ FILE\&dDocName $=$ PDF_CONF_P56_S6_01_CHO_V\& RevisionSelectionMethod=LatestReleased\&Rendition $=$ Web.]

Derrien, M., and H. Le Gléau, 2013: Algorithm Theoretical Basis Document for "cloud products" (CMa-PGE01 v3.2, CT-PGE02 v2.2 \& CTTH-PGE03 v2.2). NWC SAF Doc. SAF/NWC/CDOP/ MFL/SCI/ATBD/01, Issue 3, Rev. 2, 87 pp. [Available online at http://www.nwcsaf.org/scidocs/Documentation/SAF-NWCCDOP-MFL-SCI-ATBD-01_v3.2.pdf.]

García-Pereda, J., 2013a: Algorithm Theoretical Basis Document for "high resolution winds" (HRW - PGE09 v3.2). NWC SAF Doc. SAF/NWC/CDOP/INM/SCI/ATBD/09, Issue 3, Rev. 2, 56 pp. [Available online at http://www.nwcsaf.org/scidocs/Documentation/ SAF-NWC-CDOP-INM-SCI-ATBD-09_v3.2.pdf.]

, 2013b: Validation report for "high resolution winds" (HRW - PGE09 v3.2). NWC SAF Doc. SAF/NWC/CDOP/ INM/SCI/VR/10, Issue 1, Revision 0, 33 pp. [Available online at http://www.nwcsaf.org/scidocs/Documentation/SAF-NWCCDOP-INM-SCI-VR-10_v1.0.pdf.]
Holmlund, K., 1998: The utilization of statistical properties of satellite-derived atmospheric motion vectors to derive quality indicators. Wea. Forecasting, 13, 1093-1104, doi:10.1175/ 1520-0434(1998)013<1093:TUOSPO > 2.0.CO;2.

Menzel, W. P., 1996: Report on the Working Group on Verification Statistics. Proceedings of the Third International Winds Workshop, EUMETSAT, CD-ROM EUM P.18, 17-19.

—- W. L. Smith, and T. R. Stewart, 1983: Improved cloud motion wind vector and altitude assignment using VAS. J. Climate Appl. Meteor., 22, 377-384, doi:10.1175/ 1520-0450(1983)022<0377:ICMWVA $>2.0$. CO 2 .

Schmetz, J., K. Holmlund, J. Hoffman, B. Strauss, B. Mason, V. Gärtner, A. Koch, and L. van de Berg, 1993: Operational cloud-motion winds from Meteosat infrared images. J. Appl. Meteor. Climatol., 32, 1206-1225, doi:10.1175/ 1520-0450(1993)032<1206:OCMWFM > 2.0.CO;2.

Shimoji, K., 2012: A study on the relationship between spatial and temporal image resolutions for AMV derivation with nextgeneration satellites. Proc. 11th Int. Winds Workshop, Auckland, New Zealand, EUMETSAT, CD-ROM EUM P.60. [Available online at http://www.eumetsat.int/website/ wcm/idc/idcplg?IdcService $=$ GET_FILE\&dDocName $=$ PDF CONF_P60_S2_07_SHIMOJI_V\&RevisionSelectionMethod= LatestReleased\&Rendition $=$ Web.]

Sohn, E. H., and R. Borde, 2008: The impact of window size on AMV. Proc. Ninth Int. Winds Workshop, Annapolis, MD, EUMETSAT, CD-ROM EUM P.51. [Available online at https://www.eumetsat.int/website/wcm/idc/idcplg?IdcService $=$ GET_FILE\&dDocName=PDF_CONF_P51_S4_18_SOHN_V\& RevisionSelectionMethod $=$ LatestReleased $\&$ Rendition $=$ Web.] 